\title{
ON A NEW FAMILY OF BIVARIATE MEANS II
}

\author{
EDWARD NEUMAN
}

Abstract. Lower and upper bounds as well as inequalities satisfied by members of a family of bivariate means, introduced recently by this author in [18], are established. In particular, optimal convex combinations bounds are obtained. Also, the Wilker and Huygens-type inequalities involving means under discussion are obtained.

Mathematics subject classification (2010): 26E60, 26A09, 26D05.

Keywords and phrases: Schwab-Borchardt mean, Wilker and Huygens-type inequalities, lower and upper bounds.

\section{REFERENCES}

[1] M. Abramowitz, A. I. Stegun, Handbook of Mathematical Functions with Formulas, Graphs, and Mathematical Tables, Ninth printing, Dover Publications Inc., New York, 1970.

[2] G. D. Anderson, M. K. Vamanamurthy, M. Vuorinen, Monotonicity rules in calculus, Amer. Math. Monthly 133 (2006), 805-816.

[3] B. A. BhAYO, J. SÁNDOR, On certain old and new trigonometric and hyperbolic inequalities, Analysis Math. 41 (2015), 3-15.

[4] M. BIERNACKI, J. KRZYŻ, On the monotonicity of certain functionals in the theory of analytic functions, Ann. Univ. Mariae Curie - Sklodowska 2 (1955), 134-145.

[5] J. M. Borwein, P. B. Borwein, Pi and the AGM- A Study in Analytic Number Theory and Computational Complexity, Wiley, New York, 1987.

[6] B. C. CARLSON, Algorithms involving arithmetic and geometric means, Amer. Math. Monthly 78 (1971), 496-505.

[7] E. NeUmAn, Inequalities for the Schwab-Borchardt mean and their applications, J. Math. Inequal. 5, 4 (2011), 601-609.

[8] E. Neuman, Inequalities for weighted sums of powers and their applications, Math. Inequal. Appl. 15, 4 (2012), 995-1005.

[9] E. Neuman, Refinements and generalizations of certain inequalities involving trigonometric and hyperbolic functions, Adv. Inequal. Appl. 1 (2012), 1-11.

[10] E. Neuman, A note on a certain bivariate mean, J. Math. Inequal. 6, 4 (2012), 637-643.

[11] E. Neuman, A one-parameter family of bivariate means, J. Math. Inequal. 7, 3 (2013), 399-412.

[12] E. Neuman, Sharp inequalities involving Neuman-Sándor and logarithmic means, J. Math. Inequal. 7, 3 (2013), 413-419.

[13] E. Neuman, On a new bivariate mean, Aequat. Math. 88 (2014), 277-289.

[14] E. Neuman, On some means derived from the Schwab-Borchardt mean, J. Math. Inequal. 8, 1 (2014), $171-183$.

[15] E. Neuman, On some means derived from the Schwab-Borchardt mean II, J. Math. Inequal. 8, 2 (2014), 359-368.

[16] E. Neuman, Inequalities and bounds for a certain bivariate elliptic mean, Math. Inequal. Appl. 19, 4 (2016), 1375-1385.

[17] E. Neuman, On two bivariate elliptic means, J. Math. Inequal. 11, 2 (2017), 345-354.

[18] E. Neuman, On a new family of bivariate means, J. Math. Inequal, 11, 3 (2017), 673-681.

[19] E. Neuman, J. SÁndor, On the Schwab-Borchardt mean, Math. Pannon. 14, 2 (2003), 253-266.

[20] E. Neuman, J. SÁndor, On the Schwab-Borchardt mean II, Math. Pannon. 17, 1 (2006), 49-59. 
[21] E. NEUMAN, J. SÁNDOR, On some inequalities involving trigonometric and hyperbolic functions with emphasis on the Cusa-Huygens, Wilker and Huygens inequalities, Math. Inequal. Appl. 13, 1 (2010), 715-723.

[22] H.-J. SeIfFERT, Problem 887, Nieuw. Arch. Wisk. 11 (1993), 176.

[23] H.-J. SeIFFERT, Aufgabe 16, Würzel 29 (1995), 87. 